\title{
LE LINGUISTIQUE ET LE LITTERAIRE QU'APPORTENT LES MANUSCRITS DE LINGUISTES ?
}

\section{THE LINGUISTIC AND THE LITERARY: WHAT DO LINGUISTS' MANUSCRIPTS TEACH US?}

\author{
Jean-Claude Coquet \\ Université Paris 8, Institut des Textes et Manuscrits Modernes \\ (ITEM, UMR 8132 CNRS/ENS), Paris, França \\ Irène Fenoglio \\ École Normale Supérieure, Institut des Textes et Manuscrits Modernes \\ (ITEM, UMR 8132 CNRS/ENS), Paris, França \\ Pierre-Yves Testenoire \\ Université Paris 3, Laboratoire d'Histoire des Théories Linguistiques (HTL), Institut des \\ Textes et Manuscrits Modernes \\ (ITEM, UMR 8132 CNRS/ENS), Paris, França
}

Résumé: Larticle qui suit est un composé de trois approches qui tentent de faire apparaittre les liens entre linguistique et littérature chez les linguistes. Langues et Lettres, linguistique et littérature, ces deux domaines ont toujours été en couple, plus ou moins fusionnel, plus ou moins distendu, voire opposé dans les méthodologies d'approche de leur objet. Qu'en est-il du point de vue génétique ? Voilà les questions que nous proposons pour cette réflexion : ouvrir les archives de linguistes, rendre compte de leurs manuscrits peut-il avoir un intérêt pour la littérature ? Si la constitution élaborative d'un texte est bien le matériau initial et processuel de la littérature, en quoi, examiner cette constitution dans le domaine linguistique apporte quelque chose?

Mots clé: linguistique; littérature; point de vue génétique; archives; linguiste.

Abstract: This article follows a composition of three approaches to try to show the links between linguistics and literature through linguists. Languages; Literature and Linguistics, these two areas have always formed a more or less fusional couple, more or less distended, opposite in the methodologies to approach their object. From the genetic point of view, how does this happen? By opening linguists' files realizing their manuscripts could be of interest to literature? If the constitution of drafting a text is an initial and procedural material of literature, in what could the examination of this constitution in the linguistic field contribute to this relationship? Keyword: linguistics; literature; genetic criticism; files; linguists. 
Archives, genèse, textualisation (I. Fenoglio)

L'article qui suit est un composé de trois approches qui tentent de faire apparaître les liens entre linguistique et littérature chez les linguistes. Langues et Lettres, linguistique et littérature, ces deux domaines ont toujours été en couple, plus ou moins fusionnel, plus ou moins distendu, voire opposé dans les méthodologies d'approche de leur objet. Qu'en est-il du point de vue génétique?

Ecrire de la littérature et écrire de la linguistique procèdent d'une même activité matrice : écrire. Ecrire c'est textualiser une pensée retenue encore sous forme de discours intérieur ou de notes plus ou moins organisées, c'est la formaliser en un texte, ou plusieurs textes, de façon à ce qu' un lecteur puisse y trouver matière : matière à lire, fantasmer, évoquer, ressentir dans le cas de la littérature, matière à comprendre le fonctionnement de la langue matériau même de toute écriture - dans le cas de la linguistique.

Le travail d'explicitation de la façon dont se génère l'écriture linguistique - bien que domaine tout à fait neuf - a déjà fait l'objet d'un certain nombre d'articles et en particulier d'un numéro entier de la revue Genesis $^{l}$. Il y était montrécomment plusieurs approches se conjuguaient et se combinaient pour comprendre comment un auteur linguiste s'y prend pour penser son objet, élaborer une notion, formuler son discours, autrement dit comment ces approches permettaient de faire la genèse d'un texte linguistique : l'analyse linguistique des manuscrits ${ }^{2}$, de tout manuscrit ; l'approche purement éditoriale de manuscrits de linguistes, comme ceux

${ }^{1}$ Genesis. Manuscrits. Recherche. Invention, $\mathrm{n}^{\circ}$ 35, Le geste linguistique (Fenoglio I. eds), Paris, PUPS, 2012. On peut signaler aussi : Modèles Linguistiques, Tome XXX-1, vol. 59, 2009 (Fenoglio I. et Adam J.-M. eds), Génétique de la production écrite et linguistique ; Fenoglio I., 2011, " Déplier l'écriture pensante pour relire l'article publié. Les manuscrits de "L’appareil formel de l'énonciation" » in Relire Benveniste. Réceptions actuelles des Problèmes de linguistique générale, Brunet E. et Mahrer R. eds, Louvain la Neuve, Academia, p. 261-302 ; Fenoglio I., 2012, "Création scripturale, genèse, édition. Manuscrits littéraires (Pascal Quignard) et manuscrits scientifiques (Émile Benveniste) ", Crítica genética y edición de manuscritos hispánicos contemporáneos. Aportaciones a una "poética de transición entre estados" (eds. Vauthier B.\& Gamba Corradine J.), Salamanca, E U S, p. 259-280 ; Semen n 33 Les notes manuscrites de Benveniste sur la langue de Baudelaire (Adam J.-M., Laplantine C. eds), Besançon, PUFC, 2012 ; Chepiga V. et SofiaE. (eds), 2014, Archives et manuscrits de linguistes, Louvain la Neuve, éd. Academia.

${ }^{2}$ Voir, pour les publications les plus récentes : Grésillon A. et Lebrave J.-L., «Linguistique et génétique des textes : un décalogue " in Le français moderne, 2008, p. 37-51 ; Grésillon A. et Lebrave J.-L., "Génétique et énonciation - mode d'emploi " in Relire Benveniste. Réceptions actuelles des problèmes de linguistique générale, Brunet E. et Mahrer R. éds, Louvain la neuve, éd. Academia, 2011, p. 43-69. 
de Saussure, par exemple ; l'analyse linguistique et sémiotique du discours linguistique publiés.

La question ici va se faire autre, pas forcément plus précise mais déplacée : ouvrir les archives de linguistes, rendre compte de leurs manuscrits peut-il avoir un intérêt pour la littérature ?

Si la constitution élaborative d'un texte est bien le matériau initial et processuel de la littérature, en quoi, examiner cette constitution dans le domaine linguistique apporte quelque chose?

Nous ne répondrons pas exactement à une telle question mais par le biais exemplaire de deux linguistes, en l'occurrence Ferdinand de Saussure et Émile Benveniste, il nous sera possible de faire apparaître des lieux de passages et des espaces d'échange.

Saussure : une même exigence face au littéraire et au linguistique (P.-Y. Testenoire)

Victime d'une " incurable infirmité à terminer même la plus insignifiante rédaction dès qu'il s'agit de l'envoyer à l'imprimerie $»^{4}$, Ferdinand de Saussure a beaucoup écrit mais peu publié. L'œuvre publiée de son vivant est restreinte - guère plus de 600 pages -, austère et érudite. Entièrement consacrée à la linguistique historique et comparée des langues indo-européennes, elle ne laisse en rien présager un intérêt spécifique pour la littérature. Quelle ne fut donc pas la surprise lorsqu'en 1964 Jean Starobinski révéla l'existence du travail de Saussure sur les anagrammes. La publication d'extraits de cahiers d'anagramme dans ses articles, réunis ensuite dans les Mots sous les mots, assura à cette recherche son heure de gloire. Les textes de Saussure suscitèrent un engouement dans des domaines variés, inspirant des linguistes (Jakobson, Milner), des sémioticiens (Kristeva), des théoriciens de la littérature (Barthes, Riffaterre) des psychanalystes (Lacan, Irigaray), des philosophes (Derrida, Faye, Baudrillard)... Fondée sur une part infime

\footnotetext{
${ }^{3}$ Voir par exemple : Neveu F., 2008, « Réflexions sur la forme du discours linguistique », CMLF 2008, http://www.linguistiquefrancaise.org/index.php?option=com

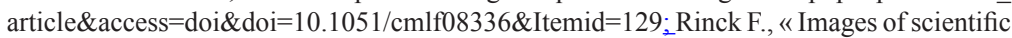
activity through the research article : a comparison between linguistics and literary studies » in Flottum K. and Korsnes O., Academic Prosa 3, University of Bergen, 2005, p. 76-86 ; Valette M. (2006). « La genèse textuelle des concepts scientifiques. Etude sémantique sur l'œuvre du linguiste Gustave Guillaume », Cahiers de lexicologie 89, p. 125-142.

${ }^{4}$ Lettre de Saussure à l'épouse de Louis Havet (Redard Georges, 1976, « Ferdinand de Saussure et Louis Havet », Bulletin de la Société de Linguistique de Paris 71, p . 341).
} 
des textes de Saussure disponibles à la Bibliothèque de Genève, cette première réception perçut un antagonisme entre le travail des anagrammes et le Cours de linguistique générale, incarnation du paradigme structuraliste qu'il s'agissait alors de contester. L'intérêt pour les anagrammes s'émoussa dès les années 80 mais ces interprétations eurent une influence durable : dans le domaine littéraire par exemple, les anagrammes saussuriens sont, via la théorie des paragrammes de Kristeva et de Riffatterre, une des sources du concept moderne d'intertexte. Plus de quarante ans après, la prise en compte de l'ensemble des manuscrits de Ferdinand de Saussure permet de porter sur ces papiers, encore largement inédits, un autre regard.

Les manuscrits consacrés à la littérature constituent environ un tiers des quelques 12500 feuillets manuscrits autographes du linguiste aujourd'hui conservés. Si certains relèvent d'une démarche strictement philologique établissement, traduction ou commentaire de textes - la majorité d'entre eux construisent une réflexion théorique sur la création littéraire et, à ce titre, dessinent une poétique. La recherche des anagrammes, à laquelle est dévolue une centaine de cahiers, forme la majorité de ces manuscrits. Cette recherche menée entre 1906 et 1909 s'inscrit cependant dans un vaste réseau d'autres travaux - sur la versification des poèmes homériques, des vers saturniens latins et du Véda - qui la précède, la suit ou l'accompagne ${ }^{5}$. L'intérêt pour les littératures anciennes, dominant chez Saussure, n'est par ailleurs pas exclusif : sont aussi conservées un ensemble de notes pour un cours de versification française qu'il dispense pendant une dizaine d'années (1900-1909) ou pour l'évaluation de poèmes contemporains en tant que membre de jury d'un prix littéraire de l'Université de Genève (prix Hentsch 1902). Ces notes manuscrites, écrites sur une période de vingt ans, ne forment pas un ensemble homogène; elles permettent néanmoins de dégager plusieurs constantes dans la démarche poéticienne du linguiste.

La première, et non des moindres, est qu'elle repose sur la prise en compte d'une diversité de langues et, tout particulièrement, des langues qui sont historiquement au cœur du projet du comparatisme linguistique. De ce point de vue, l'entreprise poéticienne de Saussure ne peut être disjointe de son activité de spécialiste de linguistique historique et comparée. Ainsi l'hypothèse des anagrammes formulée à l'été 1906 est directement issue de questionnements métriques sur les vers saturniens, ces vers latins archaïques dont l'analyse résiste aux mètres de la versification gréco-latine. C'est en cherchant à résoudre cette énigme métrique que Saussure envisage l'existence d'un principe de composition reposant sur des règles de distribution des

${ }_{5}^{5}$ Cf. Testenoire Pierre-Yves, 2013, Ferdinand de Saussure à la recherche des anagrammes, Lambert-Lucas, Limoges. 
phonèmes à l'intérieur des vers. Après avoir supposé que ces règles obéissent à des principes arithmétiques - les phonèmes devraient se répondre deux à deux dans les vers -, Saussure découvre que la structure phonique des poèmes s'organise autour d'une unité lexicale présente ou sous-entendue dans le vers qu'elle reproduirait : c'est l'hypothèse de l'anagramme. Cette hypothèse est vérifiée dans les corpus latin (des vers saturniens à la poésie néo-latine), grec (les poèmes homériques et la poésie lyrique), sanscrit (le Véda) et germanique (le Hildebrandslied et le Nibelungenlied). Ce n'est qu'après l'examen de ces quatre traditions poétiques différentes que Saussure en vient à postuler l'anagramme comme un principe commun :

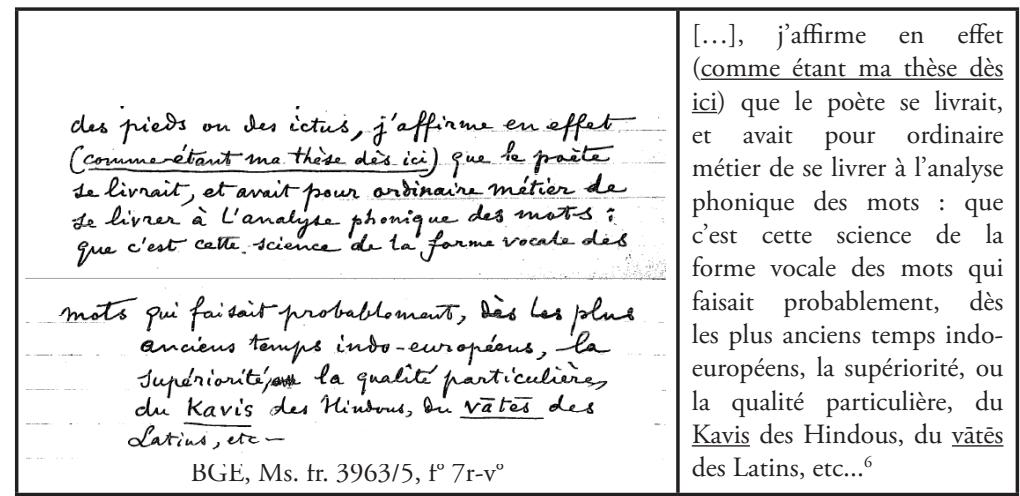

La recherche des anagrammes a donc pour objet l'activité poétique conçue comme " cette science de la forme vocale des mots » et elle poursuit les modalités de sa réalisation et de sa transmission depuis la plus haute antiquité à travers les textes, versifiés ou non, de différentes langues, à différentes époques, et dans différents genres. Loin du héros théorique que la réception a voulu un temps construire, le Saussure des anagrammes ancre ainsi sa réflexion dans l'étude comparée des productions poétiques attestées dans une pluralité de langues. Sa démarche ne s'abstrait jamais de l'historicité des textes et des cultures.

L'empirisme de la démarche s'accompagne d'un second trait : son caractère inductif. Dans ses recherches poétiques, Saussure procède comme pour la formulation de principes généraux en linguistique ou en sémiologie. Il ne part des grandes catégories - la langue poétique, le vers, les genres littéraires... - mais au contraire des faits poétiques qui lui permettent d'interroger ces catégories. De même que ses écrits théoriques effectuent

${ }^{6}$ Ferdinand de Saussure, Anagrammes homériques, éd. Pierre-Yves Testenoire, Limoges, Lambert-Lucas, 2013, p. 390. 
un travail de sape sur les " réalités trompeuses » de l'analyse linguistique "puisque nombre de linguistes ont créé des fantômes auxquels ils se sont attachés " (SAUSSURE, 1968, p. 247), de même ses manuscrits de poétique engagent un examen critique des concepts de l'analyse littéraire. La réflexion sur la notion "vers » est à ce titre exemplaire. Saussure ne cesse de rappeler dans ses manuscrits que l'analyse d'un vers ne se réduit pas à la réalisation d'un schéma métrique mais doit considérer le jeu des unités linguistiques qui le composent. En vertu de ce principe « oublié par laplupart ", écrit-il,

[...] la question est <depuis le commencement> de savoir dans quelle position se trouvent les mots vis-à-vis du vers ; car sỉil n'y avait pas cette question, cela prouverait qu'en alignant les mots d'une manière quelconque on tombe toujours sur un schéma métrique, et qu'il n'y a donc ni difficulté à faire un vers ni intérêt (Bibliothèque de Genève (BGE), Ms. fr. 3970/f, fo 8).

Questionnement de principe, donc, sur ce que c'est qu'un vers et pour lequel Saussure propose dans ses notes pour son cours de versification française une méthode originale :

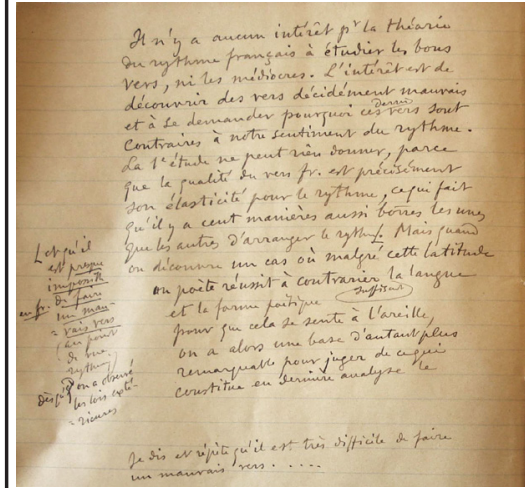

Il n'y a aucun intérêt pr la théorie du rythme français à étudier les bons vers, ni les médiocres. L'intérêt est de découvrir des vers décidément mauvais et à se demander pourquoi ces $<$ derniers $>$ vers sont contraires à notre sentiment du rythme. La le étude ne peut rien donner, parce que la qualité du vers fr. est précisément son élasticité pour le rythme, ce qui fait qu'il y a cent manières aussi bonnes les unes que les autres d'arranger le rythme <et qu'il est presque impossible en fr. de faire un mauvais vers (au point de vue rythmique) dès qu'on a observé les lois extérieures $>$. Mais quand on découvre un cas où malgré cette latitude un poète réussit à contrarier <suffisamment> la langue et la forme poétique pour que cela se sente à l'oreille, on a alors une base d'autant plus remarquable pour juger ce qui constitue en dernière analyse le

Je dis et répète qu'il est très difficile de faire un mauvais vers... 
Aussi Saussure propose-t-il à ses étudiants d'étudier pourquoi un vers comme "Je vais à l'Université " est " un très bon vers de 8 syllabes " et pourquoi "Princes et marquis des buissons " est " un mauvais vers " (BGE, AdS 379/9, f 9v-10). C'est négativement qu'il entend déterminer ce qui fait la poésie - Saussure ne parle évidemment pas de poéticité ou de littérarité et qui échappe aux règles de la versification. L'enjeu est similaire à celui des anagrammes : dégager par-delà les règles explicites de la composition d'un poème les principes de sa création. Dans ses écrits de poétique, comme dans ceux de linguistique, Saussure se veut dans l'homme des fondements.

En définitive, ce qui faitl'intérêt des manuscrits de poétique de Saussure, indépendamment des interprétations divergentes d'hier ou d'aujourd'hui ${ }^{7}$, c'est qu'ils proposent une attitude critique, une épistémologie. Saussure aborde la littérature en tant que linguiste et sa démarche est empreinte de la même réflexivité qu'il adopte vis-à-vis de la langue.

\section{Le linguiste et la littérature (J.-C. Coquet, I. Fenoglio)}

Benveniste s'intéressait à la littérature. Quelques points de repère et plusieurs documents dans ses archives en attestent. Dès 1924 - il avait 22 ans, était déjà licencié et agrégé de grammaire et 3 ans seulement le séparaient de sa nomination à l'EPHE pour succéder à Meillet comme directeur d'études - on peut lire de lui un compte rendu des Cahiers de Malte Laurids Bridge de Rainer Maria Rilke ${ }^{8}$

Rilke vit donc sous le signe de l'effroi : l'angoisse est le lieu des sensibilités qui ne comprennent pas. De là cette voix chuchotante, ce tremblement mal dominé. Pourquoi ce masque qui cache l'être ? Ou alors un être ne se dissimule-t-il pas derrière le masque des choses ? Voilà le dilemme qui le

\footnotetext{
${ }^{7}$ Pour donner une idée de la diversité des interprétations actuelles des anagrammes saussuriens, on peut mentionner trois publications récentes : l'une les interprète à la lumière des théories cognitives sur la lecture (Bravo Federico, 2011, Anagrammes. Sur une hypothèse de Ferdinand de Saussure, Limoges, Lambert-Lucas), une autre selon le prisme de l'anthropologie historique du langage de Meschonnic (Bédouret-Larraburu Sandrine et Prignitz Gisèle (éds.), En quoi Saussure peut-il nous aider à penser la littérature?, Pau, Presses Universitaires de Pau et des Pays de l'Adour, 2012), la troisième propose une lecture plus traditionnellement cryptologique du travail saussurien (Heller-Roazen Daniel, Dark Tongues. The art of rogues and riddlers, New York, Zone Books, 2013).

${ }^{8}$ Compte-rendu paru dans le numéro 1 de la revue Philosophies, du 15 mars 1924. Ce numéro est publié en " Hommage à Miguel de Unamuno ", avec des auteurs comme Max Jacob, Jules Superveille, Jean Cocteau, Philippe Soupault, P. Drieu la Rochelle. Benveniste, outre son compte-rendu de Rilke, figure parmi les signataires de cet hommage.
} 
déchire et fait naître ses angoisses. Mais cette terreur n'est pas saisie et décrite à l'instant de la crise, elle est incorporée aux incidents de la vie la plus banale, elle y circule comme un philtre au lent cheminement.

Tout de suite après ou pendant la fin de cette guerre à laquelle il a payé un lourd tribut, Benveniste écrit un texte assez surprenant et éminemment littéraire, intitulé "L'eau virile " ${ }^{9}$, et dont l'ouverture pointe immédiatement le point de rencontre entre la langue et la fiction :

Dans une représentation animée et dynamique des éléments, il se constitue toujours des oppositions, non pas seulement d'un élément à l'autre, mais d'un aspect à l'autre du même élément. L'imagination, docile à une suggestion qui émane de la matière, tend à dissocier en figures contrastées et de sexe opposé des notions que la raison tient pour simples et permanentes. La langue, les légendes témoignent de cette dualité, que les poètes réinventent chaque fois et d'autant plus sûrement que leur expression est plus authentique.

Partant d'une référence à Bachelard, L'eau et les rêves, il cite, dans ce texte très personnel, Lawrence, Claudel, Melville, Balzac, Lautréamont pour évoquer une métamorphose de la mer en océan, une " transposition, valorisation de la mer qui, même au repos, devient mâle ". Ce texte se termine par une exclamation que l'on peut supposer très intime :

Melville retrouve intuitivement la plus profonde et sans doute la plus nécessaire figuration de la mer. Elle n'est que superficiellement féminine ; même dans ses rares moments trompeurs de calme, sa puissance insondable et sa violence latente la montrent virile. On l'incarne toujours en un dieu, immémorial, élusif et solitaire, le Vieux de la Mer. Lautréamont l'invoquait justement : «Vieil Océan, ô grand célibataire!

"Solitaire " et " célibataire ", voilà deux thèmes qui retiennent l'attention de Benveniste, comme s'il s'agissait d'énoncer le mode de vie le plus convenable à la réussite de tout projet de connaissance. La règle est valable aussi bien pour le linguiste que pour l'écrivain, qu'il soit romancier, poète ("Me voilà libre et solitaire! ", écrivait Baudelaire dans Les Fleurs du mal) ou philosophe. En témoigne cette citation de Kierkegaard que l'on retrouve dans ses papiers : "Chaque fois que l'histoire du monde fait un pas important en avant et poursuit une passe difficile, s'avance une formation de chevaux de renfort : les hommes célibataires, solitaires, qui ne vivent que

${ }^{9}$ Émile Benveniste, «L'eau virile ", parue dans la revue Pierre à feu - Provence noire, n ${ }^{\circ} 1$, SaintPaul de Vence, éd. Maeght, 1945, p. 75-77. 
pour une idée $»^{10}$

Parmi les nombreux documents d'archive du linguiste on est tombé sur ce poème ${ }^{11}$ ou ébauche de poème de la main de Benveniste perdue dans d'autres feuillets très "sérieux ", un poème sur la pluie griffonné sur un long morceau de papier, au fil de la plume, il n'est pas le seul écho d'une écriture intime, quelques autres se trouvent au détour de tel ou tel document d'archive :

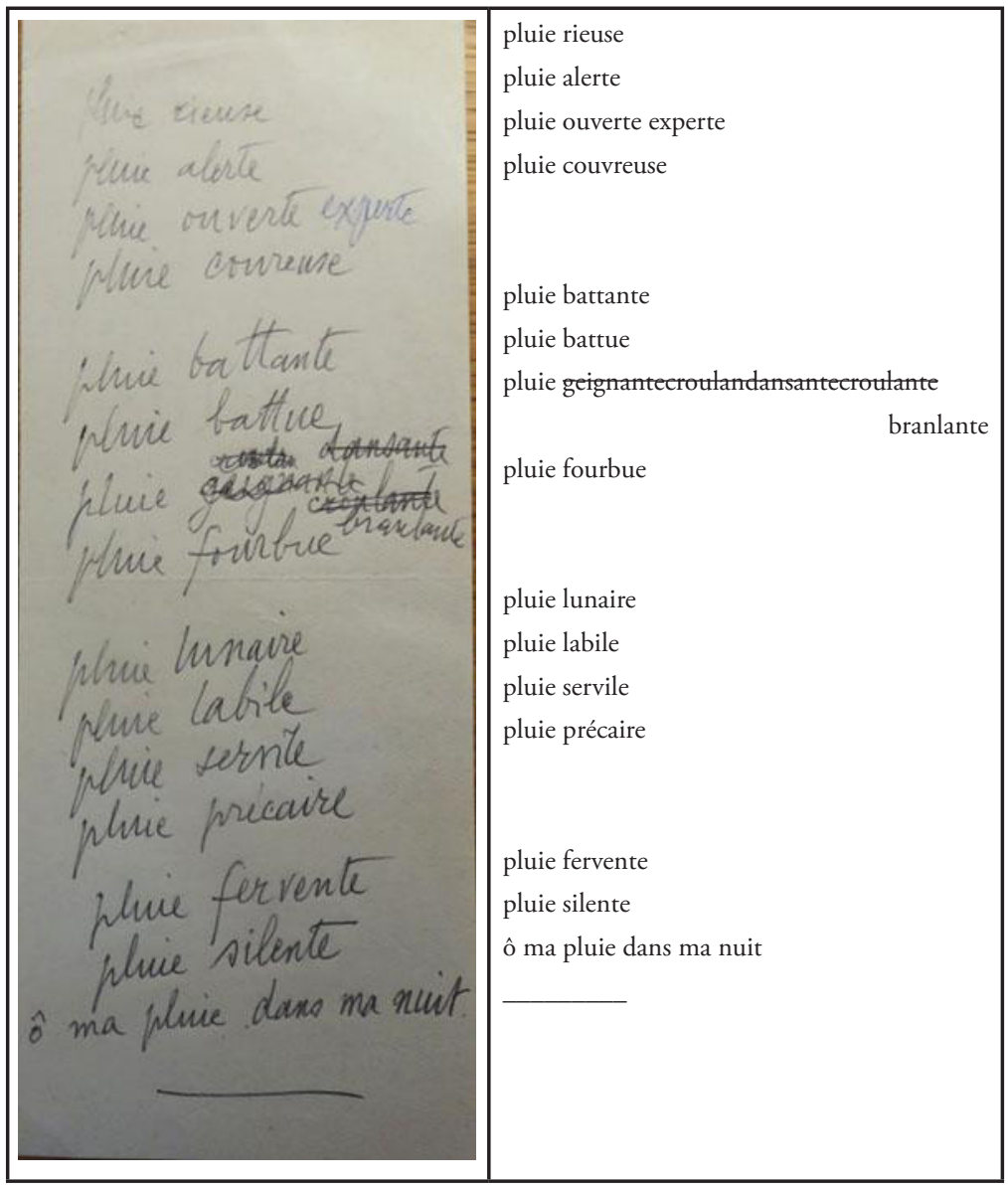

${ }^{10}$ Repris en épigraphe dans Émile Benveniste Dernières leçons, collège de France 1968 et 1969 , texte établi par J.-C. Coquet et I. Fenoglio, Paris, EHESS-Gallimard-Seuil, 2012, p. 41.

${ }^{11}$ Feuillet manuscrit conservé au Collège de France, fonds Émile Benveniste, cote : CDF 28/18. 
Il ne s'agit pas ici de juger de la valeur littéraire de ce texte, il s'agit de montrer que le geste d'écriture du linguiste s'exerce en toutes formes et que la rigueur jamais prise en défaut du très sérieux linguiste n'empêche pas l'adresse à la littérature. Si ce texte ne se terminait pas sur cette note intime : "Ô ma pluie dans ma nuit ", nous pourrions voir dans l'ensemble des propositions une suite paradigmatique exerçant un substantif à un attribut.

Pour cette sorte d'hymne à la "pluie ", Benveniste a choisi la construction en une strophe composée de trois quatrains et d'un tercet. Selon la versification classique, il fait se succéder rimes embrassées, rimes croisées, de nouveau, rimes embrassées, puis rime plate et rime intérieure. Qui a lu les Notes sur le langage iconique de Baudelaire ne sera pas surpris de la place accordée à l'échophorie, comme disent les linguistes et, peut-être, les stylisticiens. Les deux mots, "pluie » et " nuit ", qui entrent en résonance par l'intermédiaire de la rime intérieure, sont "agencés à neuf " ${ }^{12}$. Ils nous font accéder à «l'autre monde », comme dit Benveniste ${ }^{13}$, ce monde évoqué à la première personne dans le dernier vers (" ô ma pluie... ») est celui dont le poète est le seul à avoir fait l'expérience. Ce sont des "rapports d'être " ${ }^{14}$ " à chaque fois particuliers ${ }^{15}$ qui ne concernent que le corps en contact avec le monde et non le "sujet ", instance cognitive. Ce point est essentiel. Il ne s'agit pas de mettre en rapport l'idée de " pluie » avec celle de " nuit ». Il s'agit de traduire (Benveniste dit aussi " translittérer ») une expérience singulière, celle du corps propre : c'est du lien perçu par mon corps entre " ma nuit » et " ma pluie " (qui a déjà été qualifiée quinze fois) qu’il est question. Expérience du sensible, donc, expérience tout à la fois linguistique et phénoménologique, à la manière de Merleau-Ponty, réfléchissant sur "La phénoménologie du langage " (ce texte de 1951 a été conservé par Benveniste dans sa bibliothèque), et non expérience de pensée.

Les références littéraires ne manquent pas dans les articles de Benveniste. Il faudrait déjà évoquer tous les textes anciens grâce auxquels, souvent, il explicite l'accès qu'ils permettent à une langue aujourd'hui perdue. Mais si l'on parcours les Problèmes de linguistique générale, plus accessibles, on voit aussi les références plus modernes; Benveniste y utilise des exemples littéraires ou renvoie à des écrivains ou poètes. Ainsi, dans l'article "La blasphémie et l'euphémie ", il renvoie à Artaud et Bataille.

${ }^{12}$ BnF, Pap Or 0429, env. 22, f²54 (Benveniste Émile, Baudelaire, texte édité par Chloé Laplantine, Limoges, éd. Lambert-Lucas, 2011, p. 542).

${ }^{13}$ Id., env. 18, f 185 (Ibid: 402).

${ }^{14}$ Id., env. 18, f 182 (Ibid. : 396).

${ }^{15}$ Id., env. 12, f 55 (Ibid. : 134). 
Dans les notes manuscrites de "L'appareil formel de l'énonciation " il cite Verlaine. Mallarmé est cité dans "Sémiologie de la langue » et dans bien d'autres articles d'ailleurs.

Roland Barthes, dont le travail s'est toujours tenu à la croisée de la linguistique et de la littérature et qui, deux ans avant la mort du linguiste, publie ce si bel article "Pourquoi j'aime Benveniste ${ }^{16}$ lui écrivait déjà, dans une lettre, le 4 mars $1969^{17}$ :

Je ne suis pas sûr que vous ayez reçu le numéro de Langages sur « Linguistique et littérature ". C'est un numéro bien imparfait, où je ne cesse de regretter votre absence [...]. Ce m'est surtout une occasion de vous dire combien j'ai été heureux que vous acceptiez de présider l'Association internationale de Sémiologie et combien nous sommes nombreux (je le vois par mes étudiants) à être attachés à vos travaux. J'espère beaucoup que vous pourrez accepter, un jour prochain, de nous donner un texte concernant la littérature.

Barthes conserve la dénomination "sémiologie " à laquelle il est habitué, mais l'association que Benveniste aurait dû présider si la maladie ne l'en avait empêché, se dénommait "Association internationale de sémiotique $»^{18}$.

Nous n'aurons jamais de texte de Benveniste sur la littérature mais nous pouvons lire de nombreuses notes du linguiste concernant le discours poétique s'appuyant essentiellement sur des exemples tirés de Baudelaire et éditées en 2011 par Chloé Laplantine. Ces notes ont été écrites en vue d'un article " promis ", très vraisemblablement, puisque nous trouvons dans une de ces nombreuses listes auxquelles Benveniste était habitué, intitulée "Articles promis ", la mention "Langages (la langue de Baudelaire) " ${ }^{19}$ d'où l'on peut déduire que Benveniste préparait un article dont la poésie de Baudelaire constituerait le corpus d'appui. Est-ce à cet « article promis » que Barthes faisait allusion dans sa lettre de 1969 ? Il y a des chances. C'est ce corpus de notes très nombreuses et diverses, non rédigées, que nous allons examiner maintenant. Car si Benveniste, le savant en langues nombreuses, archaïques, anciennes et modernes, rigoureux chercheur en grammaire comparée et en linguistique générale, s'intéressait à la littérature, la lisait et

\footnotetext{
${ }^{16}$ Roland Barthes, "Pourquoi j’aime Benveniste ", La Quinzaine Littéraire, 16 au 30 avril 1974, p. 3.

${ }^{17}$ Lettre de Roland Barthes à Émile Benveniste, du 4 mars 1969. Conservée au Collège de France, CDF 28/12

${ }^{18}$ Voir Émile Benveniste Dernières leçons, op. cit., p. 12 et 44, note 3)

${ }^{19}$ Fonds Émile Benveniste du Collège de France, CDF 28/18
} 
pouvait la commenter, son objet était d'en comprendre les effets; pour cette compréhension il s’adressait à la linguistique.

\section{Le littéraire pour la linguistique (I. Fenoglio)}

À ouvrir la prodigieuse chemise contenant les notes de Benveniste sur le discours poétique, on reste impressionné. Ce dossier est d'une grande importance, en lui-même et en rapport avec les autres écrits et manuscrits de Benveniste. Nous y repérons, le fil rouge d'une recherche en cours, parfois fragile, parfois instable mais se précisant ici ou là avec force grâce à une multitude de remarques et d'exemples tirés de textes de Baudelaire. L'examen attentif et la lecture de ces notes montre que Benveniste cherchait à établir une spécification linguistique et phénoménologiquedu discours linguistique ${ }^{20}$.

Benveniste n'analyse pas Baudelaire en "littéraire ", ni en stylisticien, il cherche - en linguiste, en linguiste fondateur de la perspective énonciative, et donc en linguiste du discours - à comprendre ce qui distingue l'« écrit poétique " de tout autre écrit ; autrement dit, il se pose la question : dans l'usage de la langue, dans l'exercice de l'écriture, qu'est-ce qui, linguistiquement, détermine l'effet poétique, étant entendu que le premier effet spécifique est l'émotion :

Articulation de l'étude [... Il faut donc ressaisiret amener au jour ces thèmes majeurs, trahis ou énoncés par certains termes-clefs [...]Alors vient l'étude des procédés par lesquels le poète arrange/assure l'expression de ce qu'il veut communiquer, c'est-à-dire de son émotion (BnF, Pap Or 0429, env. 14, f 3 (Benveniste, Baudelaire, 2011, p. 189).

Lecteur et admirateur de Baudelaire, il se propose comme corpus sa poésie mais son objet est "Le langage poétique " et plus précisément le " discours poétique " :

Ce qu'on a fait jusqu'ici c'est l'analyse descriptive du poème.

Ce que je tente de découvrir est le mode de fonctionnement de la langue poétique (Id., env 23, f 31 (Ibid., p. 744).

${ }^{20}$ Les analyses partielles qui suivent reprennent en partie les analyses publiées dans «Fenoglio I. "Benveniste auteur d'une recherche inachevée sur "le discours poétique» et non d'un "Baudelaire ", Semen n 33 op. cit. , 2012, p.121-161 
L'ensemble des papiers de Benveniste concernant le " discours poétique » et la poésie de Baudelaire comprend 386 feuilletsdont 351 ne portent que des notes sans aucune rédaction ou avec de très partiels fragments phrasés. Seuls 35 feuillets portent des fragments rédigés pouvant, éventuellement, tenir lieu de brouillon mais nous ne trouvons aucun brouillon mis au net. Un seul feuillet s'apparente à une mise au net, il s'agit d'une dactylographie inachevée ou dont la suite est manquante ${ }^{21}$. A voir la faible proportion de feuillets rédigés par rapport aux notes de travail, on reste nécessairement circonspect : il est clair que Benveniste était loin d'avoir terminé sa réflexion, a fortiori la rédaction de son " essai $»^{22}$. La confrontation avec ses habitudes particulières de travail nous le confirme ; devant les notes concernant le "discours poétique " nous sommes devant des manuscrits dont nous ne pouvons dire l'état exact d'avancée par rapport à ce que le scrupuleux Benveniste aurait rédigé, a fortioripublié. Il est donc impensable, en l'état actuel du dossier sur le " discours poétique " - le titre porté par le seul feuillet mis au net et dactylographié existant dans le dossier a pour titre : Le discours poétique-, d'établir un texte. Il n'empêche, la lecture de ces notes est stimulante.

Il est possible, devant cette masse de feuillets, d'opérer un premier partage entre les notes constituant des relevés de données sur les textes de Baudelaire, et les notes réflexives.

$3 / 4$ Pour les notes constituant des relevés de données, nous pourrions, par exemple, indiquer cette longue note rédigée sur 4 feuillets $^{23}$, intitulée "Fondements de la poétique baudelairienne ", dans laquelle Benveniste explicite le thème des "correspondances " chez Baudelaire ; ou bien, par exemple, ce type de notes beaucoup plus élémentaires ${ }^{24}$ :

$\begin{array}{lll}\text { Souvenir } & \begin{array}{l}\text { évoquer } \\ \text { revivre }\end{array} \\ & & \begin{array}{l}\text { rêve } \\ \text { songe }\end{array}\end{array}$

Benveniste parle lui-même de " données ", ou de "relevé » : "Relevé que je crois complet ${ }^{25}$ et : "J'ai abandonné ce relevé qui ne semble pas

\footnotetext{
${ }^{21}$ Id.env. 22, f 71 (Ibid., p. 681).

${ }^{22}$ Id., env. 21, f 2 (Ibid., p. 453).

${ }^{23}$ Id., env. 22, fo 16 à 19 (Ibid., p. 571).

${ }^{24}$ Id., env. 21, fo 20 à 19 (Ibid., p. 491).

${ }^{25}$ Id., env. 14, f' 25 à 19 (Ibid., p. 233).
} 
démonstratif... $»^{26}$. Par ailleurs, il constitue des fiches pour lesquelles il met, à l'avance, un titre de rubrique, par exemple "Prégnances affectives ", "Personnalisation de choses, figuration énergisantes ", "Le temps chez Baudelaire ${ }^{27}$ etc... et qu'il remplit ensuite de données ; ainsi aussi cette fiche titrée au feutre noir : "L'emploi des temps chez Baudelaire I les Fleurs du mal ", et qui se remplit sur 18 feuillets. Plusieurs titres de fiches semblables au feutre noir avec des données en différentes couleurs d'encre, permettent de constater ce procédé.

- Pour ce qui est des notes réflexives, il faudra faire un nouveau partage entre

- les notes réflexives directement liées à Baudelaire, comme la note suivante, extrêmement importante car elle dit exactement l'usage qui est fait de Baudelaire par Benveniste ${ }^{28}$ :

La poésie a ici le visage de Baudelaire ; je parle d'elle ou de lui, sans pouvoir les distinguer. La poésie, c'est la poésie plus un certain poète puisque chaque poète a sa langue poétique

Cette note, dans son précipité, dit expressément qu'il y a eu choix de Baudelaire, comme d' " un certain poète " développant une "langue poétique " particulière, expression singulière ayant son propre système de fonctionnement et jouantle rôle de matériau d'étude pour la compréhension $d u$ discours poétique comme fait linguistique et phénoménologique.

- Enfin les notes relevant d'une tentative de formulation théorique générale pour le discours poétique qui sont les plus rédigées, comme cette note intitulée «le temps linguistique ${ }^{29}$ :

\section{Le temps linguistique}

Une distinction fondamentale à poser et à développer dans ses implications : le temps dans la mémoire / le temps hors de la mémoire

ou celle-ci de recherche purement théorique, essentielle à ce $\operatorname{dossier}^{30}$ :

Forme et sens se distribuent autrement en poésie que dans le langage

\footnotetext{
${ }^{26}$ Id., env. 14, fo 26 à 19 (Ibid., p. 235).

${ }^{27}$ Id., env. 10, f 2, 3 et 5 (Ibid., p. 95-101).

${ }^{28} I d$., env. 21, f $2 \mathrm{~V}^{\circ}$ (Ibid., p.455).

${ }^{29}$ Id., env. 9, f 10 (Ibid., p. 91).

${ }^{30}$ Id., env. 12, f 5 (Ibid., p. 139)
} 
ordinaire.

Il faut partir du niveau du signe.

Le signe poétique est, matériellement, identique au signe linguistique. Mais la décomposition du signe en signifiant-signifié ne suffit pas : il faut y ajouter une dimension nouvelle, celle de l'évocation : parrapport<qui réfere> non à la 'réalité' (concept du langage ordinaire) mais à la vision poétique de la réalité.

Hors de quelques titres en tête de relevés de données du corpus comme par exemple "Les images de la mer " ou "Le miroir " ou encore «Femme» etc. -, les titres inscrits par Benveniste sont toujours linguistiques, ils pointent un objet théorique général et interviennent le plus souvent en tête de fragments rédigés. J'ai pu relever, dans l'ensemble des 386 feuillets, 23 occurrences de titres théoriques : "Loi fondamentale de la poésie ", "Le temps linguistique ", "Structure du discours poétique ", "La poésie et la "réalitê" ", "La communication poétique ", " Eangue<Discours> poétique ", "Discours poétique ", "Le signe en poésie ", "Sonorités associatives/ évocatives ", " Rapport de coordination ", " Poésie ", "Langage poétique ", "La référence en poésie ", "<Langue> Poétique », "Principe de la 'vie du langage" ", "Poésie ", "Principe de la langue poétique ", "Langue poétique ", "Le discours poétique », "Langue poétique », " Différence de la poésie ». Et l'on peut remarquer que si " poésie " ou " poétique " sont toujours présents dans l'ensemble des titres (19 occurrences sur 23), les deux substantifs les plus présents pour supporter cet attribut sont : "discours » et " langue ».

Se demander pourquoi Benveniste a choisi ce corpus, est une vraie question d'ordre épistémologique. Baudelaire n'est pas choisi au hasard, même si existe une part subjective, un goût de lecture dans ce choix. Benveniste explicite son choix à plusieurs reprises et donne des arguments théoriques et méthodologiques.

D'une part l'œuvre de Baudelaire est immense, connue de tous; elle permet de circuler, sans quitter le discours "poétique ", entre différentes formes ou genres dont les deux extrêmes, ou les plus nommables sont la forme poème et la prose. À cette raison générale s'ajoute une perception d'éthique culturelle, civilisationnelle, pour ne pas dire historique : le souci de Baudelaire est la vérité ; être vrai passe par une perception aiguë de la "Modernité", on voit dans la note suivante l'insistance sur ce terme de vérité (trois occurrences à quoi s’ajoute "réalité vécue ») :

Baudelaire veut la vérité. Et son souci de la modernité revient à cela : être moderne, c'est être vrai, c'est abandonner les déguisements historiques, les 
fictions cosmologiques et dire l'homme dans sa réalité vécue, dans la vérité de ses sentiments et de ses expériences ${ }^{31}$.

Mais nous passons très vite à des arguments linguistiques. Baudelaire est choisi parce que son utilisation de la langue n'est pas exceptionnelle ; elles'appuie sur un ensemble linguistique structurel (syntaxe et lexique) plus " ordinaire " que celle de Mallarmé, par exemple, qui aurait d'emblée demandé des analyses d'ordre linguistique pour en stabiliser la lecture compréhensive, ce qui n'est pas le cas chez Baudelaire. Ce caractère permet de mettre en valeur le fonctionnement poétique :

Le langage iconique ne rompt pas avec le système général de la langue, il n'emploie pas d'éléments phoniques ni signifiques qui soient étrangers à la langue, et Baudelaire conserve une syntaxe qui est dans l'ensemble celle de la langue commune.

[...]

(C'est la différence entre le langage de Baudelaire et celui de poètes plus récents, à partir du dernier Mallarmé jusqu’aux surréalistes : ceux-ci veulent substituer le langage iconique au langage signifique ${ }^{32}$.

... la plupart du temps Baudelaire est encore fidèle au sens global [...]

Puis vient Mallarmé qui divorce entièrement du sens global, qui ne veut même plus de mots distincts mais que le poème soit un seul mot, une longue musique $^{33}$.

Mais l'argument le plus puissant en faveur du choix de Baudelaire est exprimé dans la note suivante, où l'on remarque immédiatement le style ramassé, télégraphique, propre aux notes de travail : Baudelaire est choisi parce qu'il s'exprime en discours, son discours poétique est du discours, matériau idéal pour le linguiste du discours :

Situation fondamentale et décisive de Baudelaire. Il est le dernier à tenir un véritable discours.

Après lui cette notion s'abolit dans la tendance Mallarmé - vers l'évanouissement profond de tout message et de l'organisation syntaxique du discours ${ }^{34}$.

Avec ce choix de corpus et, au-delà, cet ensemble de notes dessine les
${ }^{31}$ Id., env. 22, f 69 (Ibid., p. 677).
${ }^{32}$ Id., env. 6, f 5 (Ibid., p. 35).
${ }^{33}$ Id., env. 22, f 39 (Ibid., p. 617).
${ }^{34}$ Id., env. 15, f 7 (Ibid., p. 251). 
préalables et les orientations engagées d'une recherche précise : Benveniste veut comprendre quels sont les éléments linguistiques pouvant rendre compte de l'« émotion " qui caractérise l'effet poétique. Ces préalables et ces orientations se dessinent par le biais de recherches théoriques et de tâtonnements méthodologiques.

Benveniste utilise un corpus d'exemples textuels pris dans l'œuvre de Baudelaire en linguiste, non en poéticien, en linguiste qui a déjà mis au jour, et notamment dans son article "Sémiologie de la langue " le couple conceptuel sémiotique/sémantique : le sémiotique est ce qui "se reconnaît " de la langue en tout discours, le sémantique est ce qui « se comprend « du fait de l'énonciation. Comment le sémantique du discours poétique se construit en rapport au nécessaire sémiotique de la langue, telle est la question qu'il se pose. Autrement dit, comment le fonctionnement sémantique propre à toute langue mise en emploi dans un discours se spécifie en un sémantisme propre au discours poétique caractérisé par les effets émotifs qu'il provoque chez le lecteur-auditeur. Il fait place là à un niveau supplémentaire à l'intérieur de l'instance linguistique du discours où le " mot » et son " effet émotif " prendra le pas sur le signe. Ce qui l'intéresse c'est comment, linguistiquement un espace peut être déterminé pour un rapport sémiotique/sémantique serré autour d'une subjectivité d'auteur, subjectivité qui va offrir son matériau sémiotique à la compréhension sémantique finale.

Ce sont donc en réalité deux attitudes opposées. Elles se séparent sur un point essentiel à mettre en lumière : sur ce que j'appellerai la grammaire sémantique (ou poétique ?) [...] Il faut insister, pour la bien définir, sur l'originalité de la grammaire poétique de Baudelaire ${ }^{35}$.

C'est bien ce qu'il spécifie dans la note suivante ${ }^{36}$ :

Poésie

La poésie est du langage chargée d'émotion. Des mots organisés rythmiquement (William Carlos williams, Paterson cité dans Critique $\mathrm{n}^{\circ}$ 235 (déc. 1966) p. 1008-9.

En effet ce sont là deux critères essentiels : $1^{\circ}$ ) organisation rythmique, cadre formel préalable $-2^{\circ}$ ) langage chargé d'émotion - ordre sémantique spécifique.

Il se peut, c'est du moins ce que je pense après avoir circulé dans

\footnotetext{
${ }^{35}$ Id., env. 22, f 67 (Ibid., p. 673).

${ }^{36}$ Id., env. 17, f 17 (Ibid., p. 301).
} 
l'ensemble des manuscrits (ceux du "discours poétique " et ceux relatifs à l'article "Sémiologie de la langue " travaillés en même temps : 1967 et 1968) que penser le discours poétique ait contribué à engager Benveniste dans cette distinction théorique essentielle sémiotique/sémantique. En effet, le sémiotique,se " reconnaît " de la même façon dans le langage ordinaire et dans l'usage poétique du langage ;lesémantiquequi se constitue dans la syntagmation phrastique va se construire, lui, sur des composés différents : notions, intentés conceptuels pour le " langage ordinaire ", images, intentés émotionnels pour le "discours poétique " :

Il faut donc distinguer la compréhension liée à la connaissance de la langue, à l'emploi correct des signes conceptuels à l'usage commun, et la perception (émotion) liaisons inédites de mots chargés d'évoquer l'expérience intime ${ }^{37}$.

Ces mots se suivent ; ils se combinent et composent des figures neuves. Ici vaut l'observation profonde de Saussure sur la consécutivité comme principe fondamental (Anagrammes de F. de S. Merc de Fr., 1964, p. 254) ${ }^{38}$.

Nous sommes bien là au cœur d'une réflexion de fond sur le fonctionnement du langage et sur sa structuration linguistique contrainte (les signes puis les mots de la langue) mais ouverte (le conceptuel et l'émotion).

En guise de conclusion (I. Fenoglio et P.-Y. Testenoire)

Les recherches de Saussure et de Benveniste sur la littérature innervent leurs théories linguistiques. De même que le travail sur le discours poétique a vraisemblablement participé à l'élaboration du couple sémiotique/ sémantique, la recherche des anagrammes a contribué à forger des concepts saussuriens. Ainsi c'est dans l'analyse inlassable de centaines de vers que Saussure théorise le principe de linéarité comme " condition fondamentale de tout mot $"{ }^{39}$. Cette découverte, inscrite dans un cahier d'anagramme où l'on trouve les seules occurrences connues de l'adjectif linéaire dans les manuscrits du linguiste, prouve l'importance du travail sur la poésie pour la genèse de certains des concepts développés dans les cours de linguistique

\footnotetext{
${ }^{37}$ Id., env. 22, f 32 (Benveniste, 2012, p. 603).

${ }^{38}$ Id., env. 22, fo 42 (Benveniste, 2012, p. 623).

${ }^{39}$ BGE, Ms. fr. 3963/1, fo $19 \mathrm{v}$.
} 
générale ${ }^{40}$.

Si les intérêts de Saussure et de Benveniste - l'intérêt manifesté " professionnellement ", scientifiquement - n’ont jamais quitté le domaine de la linguistique, ils ont néanmoins laissé des traces certaines de la pertinence d'aller du côté de la littérature pour comprendre un fonctionnement linguistique. C'est que les deux linguistes savaient écrire, aimaient écrire. Benveniste loue chez Saussure son " constant bonheur d'expression " ${ }^{41}$, Meillet "sa langue si pure et si nette ${ }^{42}$, et Barthes écrit au sujet de Benveniste :

La langue que s'approprie Benveniste (puisque telle est sa définition de l'énonciation) n'est pas tout-à-fait celle des savant ordinaires, et ce léger déplacement suffit à constituer une écriture. L'écriture de Benveniste est très difficile à décrire parce qu'elle est presque neutre ; seul parfois un mot, à force d'être juste, pourrait-on dire tant la justesse semble s'accumuler en lui, brille, ravit comme un charme, emporté par une syntaxe dont la mesure, l'ajustement et l'exactitude $[. .$.$] attestent le plaisir que ce savant a pris à$ former sa phrase (BARTHES, 1974, p. 4).

A l'issue de ce parcours, rapide, elliptique, il convient de remarquer que l'approche de la littérature par nos deux linguistes se fait par le biais de la poésie. Il convient par ailleurs d'insister : si l'intérêt littéraire personnel est certain (comme il le serait pour un architecte, par exemple, ou un médecin), ils abordent tous deux, pour ce qui est de leur projet d'écriture, la littérature en linguiste, pour la compréhension linguistique.

\section{Références bibliografiques}

BARTHES, Roland. Pourquoi j'aime Benveniste. In : La Quinzaine Littéraire. 1974.

SAUSSURE, Ferdinand de. Cours de linguistique générale. Tome 1, éd. critique R. Engler, Wiesbaden, Otto Harrassowitz, 1968 (CLG/E, 1798).

\footnotetext{
${ }^{40}$ Cf. Testenoire Pierre-Yves, 2012, "La linéarité saussurienne en rétrospection », Beiträge zur Geschichte der Sprachwissenschaft,22, p. 149-170 repris dans Texto 19 - n. 2 (2014) : http:// www.revue-texto.net/index.php?id=3503.

${ }^{41}$ Benveniste Émile, 1964, "Lettres de Ferdinand de Saussure à Antoine Meillet ", Cahiers Ferdinand de Saussure 21, p. 92.

${ }^{42}$ Lefèvre Frédéric, 1925, Une heure avec... Antoine Meillet, Paris, Gallimard, p. 34.
} 